\title{
Seasonal surgery: acting at dawn
}

\section{F. M. Riegler}

Published online: 2 November 2016

(C) Springer-Verlag Wien 2016

Dear Reader,

Welcome to the present issue of European Surgery. In line with the multi- and interdisciplinary reality of general surgery and its highly challenged subspecialities, this issue of European Surgery orchestrates a broad spectrum of scientific reports including themes of plastic surgery (flaps and raps), oncology (lung cancer, apoptosis in rectal cancer), trauma surgery (abdominal gunshot trauma) and pancreatic surgery (groove pancreatitis). Finally, postdoc education (dfp) summarizes the effect of bariatric surgery in successful treatment of diabetes type 2. Taken together, the papers mirror the notion that it is highly important to outbalance the spectrum from basic science to clinical application. It remains to be questioned whether there exists a common basis for the understanding of disease and, if so, how much such an understanding may contribute to more successful management and prevention of disease.

Conceptually, disease impairs life quality via symptoms and/or the development of cancer. As a consequence, radiation- and chemotherapy plus/minus surgery contribute to impairment of the life quality of a woman or a man. Life quality directly relates to the being of an individual, in that it describes the wellor not wellbeing-based characteristics of a woman's or a man's BEING. "Essence-based reasoning” aims to direct the attention to the common basis of a perception. As such, the basis of disease is not the symptom, not the sign, but, as Bertrand Russel, Umberto Eco, Karl Popper, Ludwig Wittgenstein, Levi Strauss and Hanna Arendt et al. put it: the sign is the manifestation of the underlying cause, mechanism and patho-

\section{F. M. Riegler ( $ه)$}

Reflux Medical, Mariannengassse 10/9, 1090 Vienna, Austria martin.riegler@refluxmedical.com genesis. Let us thus go down to the source, to the well, to the stars; as the river takes the mountain to the sea and the rain brings the water to the tree.

Patient history harbours - to cover and reveal-the general basic ground of all diseases. However, modern medicine does not foster time to adequately translate this information from the patients. Therefore, a much too short take of the patient history fails to reveal the pathogenesis of all diseases. In contrast, when a physician has the time, she or he will learn a story of old, which has been well taken in the ancient Vedic Traditions (around 2000-3000 B.C.), the Sumerian Poetries (around 3000 B.C:) and the amazing world of the old Egyptian Pyramid Texts (around 2500 B.C), where

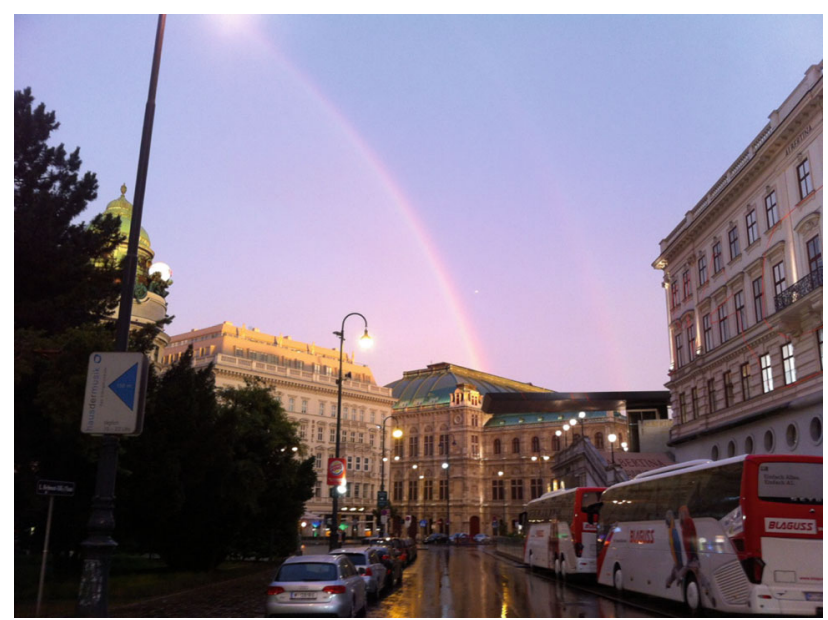

Fig. 1 The image cartoons the idea of the author that in an human being Gesamtkunstwerk organism, the rainbow spectrum of symptoms and signs can be traced back to a single cause, i. e. the lack of energy, deficit of energy, the manifestation of which is perceived in the form of different diseases. Image obtained after heavy rain and shows, next to the State Opera House, Vienna, Austria, EU, the wonderful planet earth around the summer solstice 2016 


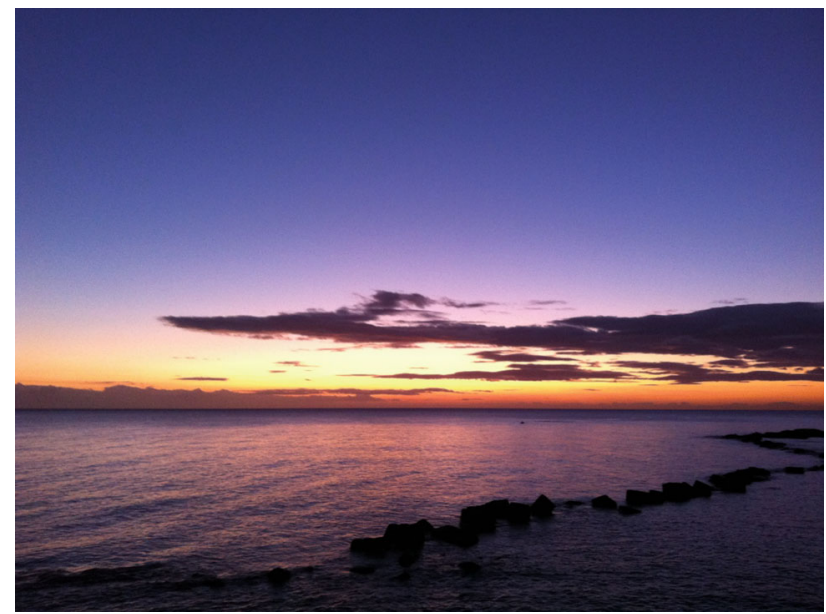

Fig. 2 The image mirrors the author's idea of "acting at the dawn of disease", when specific signs precede the full development of a disorder. This approach may, at least in theory, be suitable to foster early recognition and elimination of benign or premalignant "energy consumers" (polyps, nodes, adenomas, cysts), as outlined in the text. Image obtained during early morning dawn in Syracuse, Sicily, Italy, EU; beautiful planet earth, around winter solstice 2012

we learn a legacy of outstanding clarity, importance and relevance: there is no star without a companion, i. e. no sign without a sign maker and perceiver, no manifestation without a cause, no unpaired mechanism (tumour and so-called para-neoplasia etc.).

Taken from the molecular to the macroscopic level, the basis for any disease is the lack-of-energy state within a given system. Genetics and epigenetics (lifestyle-induced, environmental, "acquired") define the targets and forms for the manifestation of the lack-of-energy state. Depending on the targets and types of manifestations, diseases may involve a spectrum of organs from head (migraine) to feet (lumboischialgia); from ear, nose and throat (allergies), via the lungs (asthma), blood circulation system (hypertension, leukaemia) and endocrine symphony (diabetes, thyroid dysfunction) to the other extreme of the spectrum: oncology-hide the face behind the mask (Fig. 1). Here, the lack of energy causes loss of control over the repair mechanisms of a given tissue (apoptosis leathering, anoikis of epithelial tissue autumn) and paves the receptor-positive road for the mutations towards cancer development (Key Ras Tat Vam Asi). Let us get back to the patient history.

If you manage to take time, from time to time, the patients tell you about many different manifestations of lack-of-energy states and episodes which dawned prior to development of the more serious symptoms that preceded diagnosis of cancer. Become sensitive for the slight light of the dawn - the value of this information is usually underestimated. Thus, prior to the development of hypertension, diabetes or cancer, patients report that they perceived periods of anxiety, or episodes of increased sweating, tiredness, weakness and loss of libido. Next, these individuals frequently

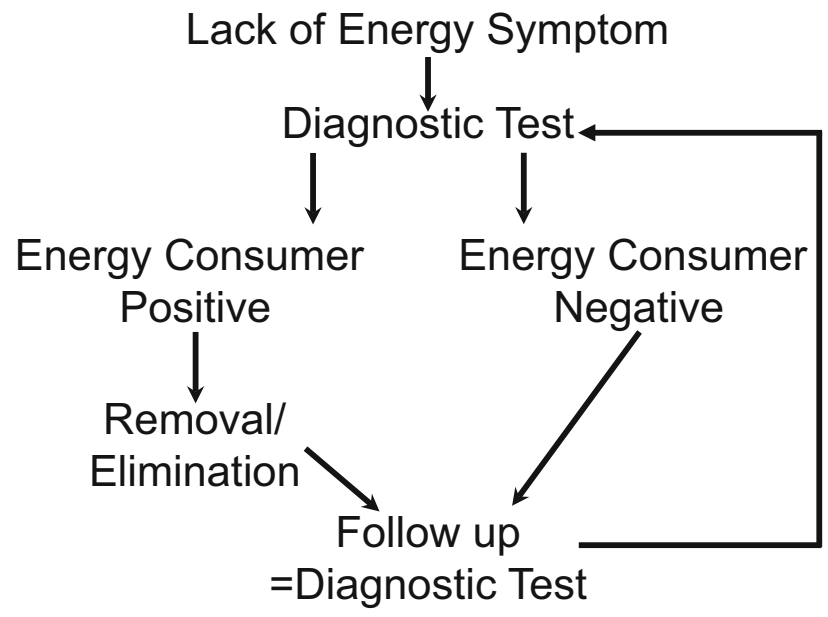

Fig. 3 Proposed algorithm to search for an "energy consumer" that causes the lack-of-energy state, which, in turn, manifests as the spectrum of symptoms, as outlined in the text. Elimination of energy consumers includes pharmacological, interventional and surgical therapies. Follow-up intervals for the diagnostic tests are timed according to the characteristics of the energy consumer (i. e. metabolic imbalance, tissue adenoma and dysplasia; or negative for metabolic imbalance, tissue dysplasiaand adenoma). Diagnostic tests include blood analysis, pharmacological, endoscopic, surgical and nuclear medicine/radiologic procedures. Arrows indicate the sequence of steps within the algorithm

report other manifestations of lack-of-energy states, including headache, increased heartrate (mainly during the night, when the continent faces the black skies of the endless universe), epigastric pain, diarrhoea and restless legs episodes. These signs refer to the deficit of energy caused by the underlying theme, i. e. the "energy consumer" (e. g. polyp, cancer?) - the reason and origin for the lack-of-energy state-since they represent major energy consumers:

1. the imbalance of metabolism,

2. premalignant lesions (polyps, adenomas, complicated cysts, aberrant ducts) and

3. cancer induce the deficit of energy state, which in turn fosters

4. genetic mutations in other organs and tissues.

As a consequence, the abovementioned symptoms arise. The impact of acting at dawn, instead of waiting until the end of the day, remains to be questioned (Fig. 2). Let us search for the energy consumer within the Gesamtkunstwerk human (Fig. 1).

Imagine you could jump out of the World Wide Web-based global village Google Facebook Twittered high-speed life, at least for several hours per week. As such, you may be able to adequately listen to the stories and poetries of your patients. You may learn: it is much harder to listen than to talk; more difficult to offer than to receive attention. Based on the above considerations you may learn to interpret the score and understand what sounds, through the spectrum 
phenomena (symptoms, signs), the presence of an energy consumer. Furthermore, this would enable you to assess tumours prior to cancer development, i. e. if you screen for the energy consumer. A large body of evidence prooves that early recognition and elimination of premalignant lesions (polyps, nodes, adenomas, aberrant ducts) contributes to cancer prevention, improvement of life quality and harbours economic benefits (less costs arising from expensive therapies). However, early detection is impaired by the fact that our screening tests are frequently applied too late, to the wrong persons and only a small portion of clients undergo these tests. It remains to be questioned how to better focus the tune.

Why not act at dawn (Fig. 2)? If you assess one of the above signs for lack of energy (tiredness, weakness, irritable bowel syndrome, gastroesophageal reflux disease), take the body of the patient and test it for all possible causes of the lack-of-energy state, screen for all possible energy consumers (skin, breast, lung, thyroid, blood, gut etc.; Fig. 3). You will have to rule out metabolic, infectious and premalignant disorders, as well as (early) cancer of to ecto- and mesodermal origin. In theory, following the diagnosis, elimination of these causes by a spectrum of methods (medical, nutrition, surgery, mental training, etc.) will contribute to reshaping the wellbeing and restoring the life quality (Fig. 3). Finally, acting at dawn may contribute to cancer prevention. However, this also needs an open mind and the sensitivity of the patients to see the doctor and tell him about the dawn signs of a disease. In turn, physicians and politicians would do better to inform and educate the population in this respect. As a consequence, tailored, personalized medicine will promote the uprising of a season in which prevention of disease will replace treatment of a disease in the future, at least in part. I am aware that such an approach will only be attractive and possible for a minority of physicians, patients and clients within the medical, pharmaceutical, food and beverages industry. But at least it should be offered to the minority who are interested in seasonal surgery. Think it over, stay tuned and foster wellbeing with harmonized positive seasonal greetings.

Acknowledgements The author thanks his patients, family, teachers, colleagues and friends for their patience and motivation to support his search for the essence of perception, which represents an integral part of medicine, surgical science, diagnosis and therapy. May they continue to spread their fruitful ideas to open the minds for essence-based reasoning, as in the olden days, as published by Peppa Pig, Heraclitus, Parmenides, Anaximander, Homer, Hesiod and many, many more. Finally, the author thanks the beauty of the planet, which takes the moon and us around the sun, all the way through space, the major source for our theme, as we are.

Conflict of interest F. M. Riegler declares that he has no competing interests. 